\title{
Mathias Mayer
}

\section{Marcel Prousts Königin von Neapel Mit einem Exkurs zu Hofmannsthal}

Marcel Proust ${ }^{1}$ musste bekanntlich einiges dafür tun, um die Aufregung zu lindern, die im Freundes- und Bekanntenkreis aufgekommen ist, wenn es um die Durchschaubarkeit biografischer Vorbilder für die eine oder andere seiner Figuren ging. Die Tatsache, dass der Autor die eigene Homosexualität von der Erzählerfigur auf andere Protagonisten gleichsam umgelenkt hat, spielt dabei eine zwar wichtige, aber nicht die entscheidende Rolle. Statt biografischem Voyeurismus kommt es darauf an, die Bewegungen, die zwischen Fiktion und Realität in beiden Richtungen verlaufen, genau zu erfassen. Besonders heikel mochte zwar das Verhältnis Prousts zu dem 16 Jahre älteren Schriftsteller Robert de Montesquiou sein, der ihn 30 Jahre davor in die Kreise des Faubourg Saint-Germain eingeführt hatte, nun aber, 1921, im Erscheinungsjahr von "Du Côté des Guermantes II" und »Sodome et Gomorrhe I«, sich in Baron Charlus hatte oder hätte porträtiert finden können. Proust betreibt einen liebenswürdigen Aufwand, sowohl direkt ihm gegenüber als auch in der Mitteilung an Dritte, dass man "unsinniger Weise« behauptete, er habe Montesquiou als Charlus dargestellt. »Das wäre um so boshafter«, versichert Proust dem Kritiker Jacques Boulanger,

als ich, der ich zwar in der Gesellschaft eine enorme Anzahl Invertierter gekannt habe, denen kein Mensch es anmerkte, niemals in den vielen Jahren,

1 In Adornos Auseinandersetzung mit dem Vorwurf "unsympathische[r] Leute« gegenüber Prousts Snobismus, "warum man für eine schon zu Prousts Zeiten ihrer realen Funktion enteignete und statistisch keineswegs repräsentative Hocharistokratie sich interessieren solle«, fällt der denkbar erhellende Satz: "Prousts Regression ist ein Stück Utopie«; vgl. Theodor W. Adorno, Kleine Proust-Kommentare. In: Ders., Gesammelte Schriften. 20 Bde. Hg. von Rolf Tiedemann, Darmstadt 1998, Bd. 11: Noten zur Literatur, S. 203-215, hier S. 209 und 211f. Indem Adorno einer »Versenkung ins Bruchstück « folgt, ebd., S. 204, umschreibt und legitimiert er die These, jede Einzeldarstellung berge »Konstellationen dessen in sich, was am Ende als Idee des Romans hervortritt«, ebd., S. 203. Weit unterhalb eines solchen Anspruchs ist der folgende Versuch situiert, der überdies den Verdacht des Biografismus auf den Plan ruft. Für instruktive Kritik und hilfreiche Hinweise danke ich für den Proust-Teil Prof. Dr. Thomas Klinkert, Zürich, für Hofmannsthal und die Königin von Neapel Dr. Konrad Heumann, Frankfurt a.M. 
seitdem ich Montesquiou kenne, ihn, weder zu Hause noch in Gesellschaft oder sonstwo, das geringste Anzeichen davon habe geben sehen. ${ }^{2}$

Wenige Wochen davor hatte er den Betroffenen selbst zu beruhigen versucht - »[i]n jedem Werk (ich spreche nicht von einzelnen Bänden, sondern vom Gesamtwerk) gibt es kaum zwei oder drei Schlüssel, und auch die öffnen nur für einen kurzen Augenblick « ${ }^{3}$-, um dann den Verdacht auf ein anderes Vorbild zu lenken, den verstorbenen Baron Doason, der die Anregung für einen intensiven Blickkontakt zwischen Charlus und dem Erzähler geboten habe, "[d]och ich habe ihn dann fallen lassen und einen viel breiter angelegten Charlus geformt, der völlig eigene Erfindung ist « ${ }^{4}$. Gleichwohl haben die Kenner ausgemacht, dass neben heiklen autobiografischen Erfahrungen Montesquiou, der Baron Doason, aber auch Jean Lorrain (mit dem sich Proust 1898 duelliert hatte) und Oscar Wilde für einzelne Züge des Baron Charlus Pate gestanden haben. Ähnlich reiche Hintergründe konnten für die Herzogin von Guermantes, für Albertine oder Robert de Saint-Loup ermittelt werden, denn, so zitiert der Biograf George D. Painter den Autor, »es gibt keinen Schlüssel zu den Personen meines Romans; oder vielmehr, es gibt acht oder zehn Schlüssel zu jeder einzelnen « ${ }^{5}$. Ein vergleichbarer Fall palimpsestartiger Überschreibungen liegt überdies in den Orten und Gegenständen des Romanzyklus vor: "Ich kann Ihnen nicht sagen, wie viele Kirchen für meine Kirche von Combray - in 'In Swanns Welt ‘ >Modell gestanden ${\text { haben. }{ }^{6}}^{6}$

Was Proust hier als - vielfach bestätigte und differenzierte - Regel seiner auch partiturartigen Schreibweise offenbart, wird, wie immer in solchen Prozessen!, dort besonders instruktiv, wo diese Regel einmal unterbrochen scheint. So wenig natürlich der Roman als grandioses Zeitund Gesellschaftspanorama es ganz umgehen konnte, die eine oder andere Person des zeitgeschichtlichen Lebens zu nennen, etwa indem die Protagonisten über sie sprechen - so etwa fallen die Namen von Claude

2 Marcel Proust, Briefe zum Werk. Ausgewählt und hg. von Walter Boehlich. Frankfurt a.M. 1977, S. 449.

3 Ebd., S. 440.

4 Ebd., S. 441.

5 George D. Painter, Marcel Proust. Eine Biographie in zwei Bänden. Frankfurt a.M. 1986, Bd. I, S. 14. - Genutzt wurde auch die neuere Biografie von Jean-Yves Tadié, Marcel Proust. Aus dem Französischen von Max Looser. Frankfurt a.M. 2008.

6 Proust, Briefe zum Werk (wie Anm. 2), S. 443f.

194 Mathias Mayer 
Debussy, Richard Strauss oder den Künstlern der »ballets russes" -, so sehr macht es doch einen Unterschied, wenn Proust einmal eine historische Person ins Personal seiner fiktiven Figuren hineinschmuggelt. Gerade die Unauffälligkeit, mit der es ihm gelingt, Gegebenheiten der Zeitgeschichte im Kontext der aristokratischen Zusammenkünfte als Teil der erfundenen Welt erscheinen zu lassen, gehört zu den fesselndsten Zügen dieses Romans. Eine in diesem Zusammenhang jedenfalls sehr bemerkenswerte Figur ist diejenige der Königin von Neapel.

Sie gehört im fünften Teil des Romans "La Prisonnière" (erschienen 1923) zu denjenigen Gästen, die Baron Charlus in die Gesellschaft eingeladen hat, die die Verdurins veranstalten. Es ist jener Abend, der für den gesellschaftlichen Absturz des Barons wesentlich verantwortlich ist, hat er doch nicht daran gedacht, dass die von ihm hinzugeladenen Gäste eigentlich ihre Worte und ihren Dank an Madame Verdurin zu richten hätten.

Einzig die Königin von Neapel, in der das gleiche edle Blut pulsierte wie in ihren Schwestern, der Kaiserin Elisabeth und der Herzogin von Alençon, fing ein Gespräch mit Madame Verdurin an, als sei sie mehr um des Vergnügens willen, diese zu sehen, als wegen der Musik und wegen Monsieur de Charlus gekommen, machte der Padrona unzählige Elogen, wurde nicht müde, sich darüber zu äußern, wie lange sie schon Lust gehabt habe, ihre Bekanntschaft zu machen, sagte ihr Komplimente über ihr Haus und sprach von den verschiedensten Dingen, ganz als sei sie bei ihr zu Besuch. Sie hätte so gerne, sagte sie, ihre Nichte Elisabeth (dieselbe, die kurz darauf den Prinzen Albert von Belgien heiraten sollte) mitgebracht; diese werde sicher sehr ihre Abwesenheit bedauern. Sie schwieg, als sie sah, daß die Musiker sich auf die Estrade begaben, und ließ sich Morel zeigen. Sicherlich machte sie sich keine Illusionen über die Gründe, weshalb Monsieur de Charlus den jungen Künstler von so viel Bewunderung umgeben sehen wollte. Aber mit der alten Weisheit einer Monarchin, in deren Adern ein Blut rollte, das zu den edelsten der Weltgeschichte gehört und den größten Reichtum an Erfahrungen, an Skepsis und an Stolz in sich aufgespeichert hat, betrachtete sie die unvermeidlichen Schwächen der Menschen, die ihr die liebsten waren - ihr Vetter Charlus zum Beispiel, dessen Mutter ebenso wie die ihre eine Herzogin in Bayern gewesen war - als Mißgeschicke, um derentwillen die Stütze, die sie ihnen gewähren konnte, für jene nur um so wertvoller war und ihnen deshalb von ihr mit um so größerem Vergnügen zur Verfügung gehalten wurde. Sie wußte, daß Monsieur de Charlus in zwiefacher Weise gerührt sein mußte, daß sie unter solchen Umständen sich hierherbemüht 
hatte. Ebenso herzensgut, wie sie sich einst tapfer gezeigt hatte, versuchte diese heldenhafte Frau, die als wahre Soldatenkönigin selbst den Schuß auf den Mauern von Gaëta gelöst hatte und die immer bereit war, sich ritterlich auf die Seite der Schwachen zu schlagen, als sie Madame Verdurin allein und verlassen dastehen sah - offenbar wußte diese nicht, daß sie der Königin keinen Augenblick hätte von der Seite weichen dürfen - auch noch den Anschein zu erwecken, daß für sie, die Königin von Neapel, das Zentrum und der Anziehungspunkt dieses Abends, um dessentwillen sie sich eingefunden hatte, die Padrona war. Sie entschuldigte sich immer wieder, daß sie nicht bis zum Schluß bleiben könne, da sie, obwohl sie sonst nur selten ausgehe, noch eine andere Soiree zu besuchen habe, und bat vor allem darum, man möge sich durch ihren Aufbruch keinesfalls stören lassen, wobei sie Madame Verdurin von Ehrungen dispensierte, deren Unerläßlichkeit dieser gar nicht zum Bewußtsein kam. (Bd. V, 328-330) ${ }^{7}$

Diese persönlich anrührende Szene, in der eine Person der Hocharistokratie sich als durch und durch menschlich beweist, gewinnt überdies tragisches und ironisches Gewicht, als Charlus am Ende des Abends meint, Madame Verdurin sich zu Dank verpflichtet zu wissen:

"Ich weiß nicht, ob Ihre heraldischen Kenntnisse ausreichen, damit Sie ganz die Bedeutung dieser Veranstaltung ermessen können, das Gewicht, das ich gestemmt, die Luftverdrängung, die ich für Sie bewirkt habe. Sie haben die Königin von Neapel gehabt, den Bruder des Königs von Bayern, die drei ältesten Pairs. Wenn Vinteuil Mohammed ist, so können wir sagen, daß wir für ihn die unbeweglichsten aller Berge versetzt haben. Bedenken Sie, daß die Königin von Neapel zum Zweck der Teilnahme an Ihrem Fest aus Neuilly gekommen ist, was sehr viel schwieriger für sie war, als die Beiden Sizilien zu verlassen, setzte er mit der Absicht ziemlich massiver Ironie trotz seiner Bewunderung für die Königin hinzu. Es handelt sich da um ein historisches Ereignis. Denken Sie daran, daß sie vielleicht seit der Einnahme von Gaëta niemals mehr ausgegangen ist. Wahrscheinlich wird man in den Nachschlagewerken als Kulminationspunkte ihres Lebens den Tag der Einnahme von Gaëta und außerdem den der Soiree bei den Verdurins bezeichnen. Der Fächer, den sie abgelegt hat, um Vinteuil besser zu applaudieren, verdient berühmter zu bleiben als der, den die Fürstin Metternich zerbrochen hat, als Wagner ausgepfiffen wurde." - "Sie hat ihn sogar vergessen, ihren Fächer", sagte Madame Verdurin, die sich im Augenblick durch die Erinnerung an die Sympathie etwas beschwichtigt fühlte, welche die Königin ihr bewiesen hatte, und zeigte dabei Monsieur de Charlus den Fächer, der auf einem Fau-

7 Alle Zitate folgen der Ausgabe: Marcel Proust, Auf der Suche nach der verlorenen Zeit. Deutsch von Eva Rechel-Mertens. 7 Teile (I-VII) in 10 Bdn. Frankfurt a.M. 1981-1984. 
teuil zurückgeblieben war. »Oh, wie rührend, rief Monsieur Charlus, indem er sich verehrungsvoll der Reliquie näherte. Er ist um so ergreifender, als er schauderhaft ist; die kleinen Veilchen darauf sind tatsächlich die Höhe." Auf seinem Antlitz kämpfte ein Zucken der Rührung mit dem der Ironie. »Mein Gott, ich weiß nicht, ob Sie diese Dinge ebenso empfinden wie ich. Swann hätte Krämpfe bekommen und wäre gestorben, wenn er so etwas sähe. Ich selbst bin mir ganz klar darüber, daß ich diesen Fächer, wie hoch der Preis auch getrieben wird, bei der Versteigerung nach dem Tode der Königin kaufen werde. Denn sicher wird alles versteigert, da sie keinen Heller besitzt«, fügte der Baron hinzu, bei dem sich grausamste üble Nachrede noch unter die aufrichtigste Verehrung mischte, obwohl beide aus zwei ganz verschiedenen Naturen kamen, die er jedoch in sich zu vereinen wußte. (Bd. V, 366f.)

Madame Verdurins Wut gegenüber dem zunehmend seniler gewordenen Baron, der seinen Liebhaber und Schützling, den zwielichtigen Violinisten Charles Morel, versucht hatte zu protegieren (und der Königin vorzustellen), entlädt sich in der massiven Aufforderung eben diesem gegenüber, sich aus der beschämenden Abhängigkeit von Charlus zu lösen. Morel setzt diesen Bruch umso schneller und provokativer in Szene, als er sich um die Hoffnung getrogen sieht, durch Charlus noch der Königin von Neapel vorgestellt werden zu können, da diese bereits die Soiree verlassen hat. Charlus wird durch die öffentliche Aufkündigung der Freundschaft durch den ihm sozial denkbar weit unterlegenen Morel zutiefst gedemütigt, seine lange Sprach- und Hilflosigkeit nimmt der Erzähler, der ihn am Ende mit König Lear vergleichen wird (Bd. VII, 246), einfühlsam wahr.

Aber neben dem Erzähler ist es noch eine andere Figur, die den beschämend bloßgestellten Baron mit ihrer Sympathie begleitet - die überraschend noch einmal zurückgekehrte Königin von Neapel, die ihren liegen gebliebenen Fächer lieber selber abholen wollte, erkennt im Nu die Peinlichkeit der Situation, lässt die aufdringliche Madame Verdurin und den ehrgeizigen Morel abblitzen und widmet sich ausschließlich dem in seinem Selbstbewusstsein gekränkten, ja gebrochenen Baron:

Als sie sah, daß diese sie nicht zu erkennen schien, sagte sie: »Ich bin Madame Verdurin, Eure Majestät erkennen mich offenbar nicht.« - »Doch, doch«, sagte die Königin und setzte dabei so selbstverständlich ihre Unterhaltung mit Monsieur de Charlus fort, mit einer so vollkommen zerstreuten Miene zudem, daß Madame Verdurin zweifelte, ob dieses in einem wundervoll beiläufigen Ton ausgesprochene `Doch, doch überhaupt an sie gerichtet war, was Monsieur de 
Charlus inmitten seines Liebeskummers das dankbar genießerische Lächeln eines Experten auf dem Gebiet hochmütiger Zurechtweisungen entlockte. Morel, der von weitem die Vorbereitungen für seine Vorstellung im Gange sah, war jetzt näher getreten. Die Königin reichte Monsieur de Charlus ihren Arm. Auch auf ihn war sie böse, aber doch nur, weil er seinen feigen Beleidigern nicht beherzter entgegentrat. Sie errötete um seinetwillen vor Scham, daß die Verdurins ihn so zu behandeln wagten. Die von einfacher Güte erfüllte Sympathie, die sie ihnen vor ein paar Stunden bezeigt hatte, und der unnahbare Stolz, mit dem sie ihnen jetzt gegenübertrat, wurzelten an der gleichen Stelle ihres Herzens. Die Königin war eine Frau voller Güte, aber sie verstand die Güte zuallererst unter der Form der unerschütterlichen Anhänglichkeit an die Menschen, die sie liebte, an ihre Familie, an alle, die ihres Blutes waren und zu denen auch Monsieur de Charlus zählte, endlich alle Menschen des Bürgertums oder auch des einfachsten Volkes, welche die zu achten wußten, die sie selber liebte, und für sie gute Gefühle hegten. Als einer mit diesen trefflichen Instinkten begabten Frau hatte sie Madame Verdurin ihre Sympathie kundgetan. Zweifellos liegt hier eine etwas enge, etwas toryhafte Form der Güte vor, die mehr und mehr aus der Mode kommt. Das bedeutet aber nicht, daß diese Güte weniger aufrichtig und weniger glühend wäre. (Bd. V, 431)

Trotz seiner bekannten Laster verteidigt die Königin den schwerbeleidigten Verwandten und verhilft ihm in sehr bewegender Weise zu einem Abgang vom Schauplatz der Niederlage, der ihm das Gesicht zu wahren erlaubt - eine der anrührendsten Szenen des Romans:

Im übrigen berühren sich die Gegensätze dabei, da ja der Edle, der Nahestehende, der schwerbeleidigte Verwandte, den die Königin zu verteidigen gedachte, Monsieur de Charlus war, also trotz seines Adels und aller der verwandtschaftlichen Bande, die ihn mit der Königin verknüpften, jemand, dessen Tugenden von vielen Lastern überlagert waren. "Sie sehen aus, als fühlten Sie sich nicht wohl, lieber Vetter, sagte sie zu Monsieur de Charlus. Lehnen Sie sich auf meinen Arm. Seien Sie gewiß, daß er Sie immer stützen wird. Seine Kraft reicht dafür aus.« Dann blickte sie stolz geradeaus (ihr gegenüber befanden sich, wie mir Ski erzählte, in diesem Augenblick Madame Verdurin und Morel) und fuhr fort: "Sie wissen, daß er vormals in Gaëta die Kanaille in Schach gehalten hat. Er wird auch jetzt für Sie ein Bollwerk sein." Und so, an ihrem Arm den Baron fortgeleitend und ohne sich Morel vorstellen zu lassen, verließ die ruhmreiche Schwester der Kaiserin Elisabeth den Salon. (Bd. V, 432f.)

Dass Madame Verdurin später diese Königin von Neapel als Spionin diffamiert (Bd. VII, 112f.), zeigt den erstaunlich politischen Charakter, der 
mit der Einschätzung dieser Figur verknüpft ist. Spricht aus Madame Verdurin der engstirnige Blick des französischen Nationalismus - denn die Königin von Neapel hat wie Charlus ein bayerisches Elternteil -, so ist die Großherzigkeit und Sympathie des Erzählers (wie des Autors) für sie umso erstaunlicher, als der Teil des Romans, in dem sie auftritt, während oder nach dem Ersten Weltkrieg geschrieben worden ist.

Aber das Erstaunlichste ist die Tatsache, dass Proust hier eine historische Gestalt so gekonnt in seine Gesellschaft der Romanfiguren eingebaut hat, dass selbst das, was aus ihrem Leben berichtet wird, etwa die mutige Verteidigung von Gaëta, einen romanhaften Anschein gewinnt. Es ist sozusagen alles historisch real, was aus der Biographie der Königin von Neapel berichtet wird, aber es wirkt wie erfunden. Indem Proust eine Art Verwandtensolidarität zwischen den Verliererfiguren stiftet, der zur Armut herabgesunkenen Königin und dem bloßgestellten, in seinem Begehren geradezu entblößten Charlus, integriert er unauffällig das Historische ins Fiktive. Im Unterschied zu den meisten Figuren des Romans ist die Königin von Neapel ohne eine Umschichtung aus der biographischen Erfahrung unmittelbar in die Romanhandlung eingebunden.

Marie Sophie Amalie wurde als Herzogin von Bayern am 4. Oktober 1841 in Possenhofen geboren, als Tochter des Herzogs Max Joseph und seiner Ehefrau Ludovika. Wie es Proust berichtet, war sie die Schwester der österreichischen Kaiserin Elisabeth. In Abwesenheit des Bräutigams wurde sie mit 17 Jahren an Kronprinz Franz verheiratet, der fünf Jahre älter war als sie und dem sie als letzte Königin beider Sizilien nach Süditalien folgte, wo die eigentliche Trauung 1859 stattfand. Sie blieb dem Land fremd, der Ehemann regierte unglücklich und erfolglos, so dass nach dem Tod seines Vaters, König Ferdinand II., die Stiefmutter Franz', Maria Theresia von Habsburg, die Regierungsgeschäfte übernahm. Der unter Garibaldi sich formierende italienische Nationalismus wandte sich auch gegen die neapolitanische Königsfamilie, die ihre letzte Zuflucht in Gaëta suchte, das unter der Obhut des Schweizer Generals Felix von Schumacher stand. Die erst 19-jährige Königin versuchte durch unerschrockenen persönlichen Einsatz, Versorgung und Ermutigung der Soldaten, die Kapitulation zu verhindern, sogar mit dem Gewehr in der Hand. Gaëta war nicht zu halten, das Königspaar konnte nach Rom entkommen, wo sie unehelich schwanger wurde, wie sie auch sonst ein 
eher skandalträchtiges Leben führte. Jahre danach brachte sie eine Tochter ihres Ehemanns zur Welt, die indes früh verstarb. Vor den Truppen, die Italien vereinigen wollten, floh das Paar 1870 von Rom nach Paris und lebte dort sehr zurückgezogen. Franz II. starb 1894, das Erbe ging an seinen Bruder, Marie zog - wie es bei Proust heißt - nach Neuilly bei Paris. Sie soll einen Spitzenladen auf dem Platz vor der Oper unterhalten und ein Gestüt für Rennpferde gegründet haben. In den 1920er Jahren kehrte sie nach München zurück, wo sie am 19. Januar 1925 starb. ${ }^{8}$ In den Memoiren von Joachim Ringelnatz findet sich folgende Anekdote:

In einem Hotel am Stachus wohnte die Königin von Neapel. Ich sah sie einmal, da sie vorm Portal aus ihrer Equipage stieg. Sie trug ein prächtiges Blumenbukett. Das schenkte sie einer ärmlich uniformierten Ritzenschieberin, die gerade dort stand, und schritt dann majestätisch ins Hotel. Das kleine, blöde Trambahnschienenweiberl sperrte wortlos den Mund auf und blickte ratlos auf die Blumen.

Die Resonanz ihres großen Mutes hat sich mehrfach niedergeschlagen, Grillparzer dichtete ein Epigramm auf sie, Benedetto Croce würdigte sie 1927 als eine Figur des alten Italien. Wie wir aus einem Brief Hofmannsthals an seinen Vater wissen, war die Königin von Neapel bei der Pariser Uraufführung jener "Josephslegende" präsent, die als Gemeinschaftswerk von Hofmannsthal, Graf Kessler und Richard Strauss die letzte Premiere vor dem Weltkrieg war - eben jenes Ballettes, das auch Marcel Proust gesehen hat und in "La Fugitive« als »überaus glanzvoll« erwähnt (Bd. VI, 322). Es besteht also Anlass zur Vermutung, dass Proust, Hofmannsthal und auch die Königin von Neapel an diesem Abend im selben Haus waren. ${ }^{9}$

\section{Exkurs über Hofmannsthal}

Bei solchen Transpositionen zwischen Realität und Fiktion ist vielleicht Hofmannsthal Proust am nächsten gekommen. Während seines Parisauf-

8 Joachim Ringelnatz, Mein Leben bis zum Krieg [1931]. In: Ders., Das Gesamtwerk in sieben Bänden. Zürich 1994, Bd. 6, S. 313 (freundlicher Hinweis von Dr. Konrad Heumann, Frankfurt a.M.).

9 Vgl. SW XXVII Ballette - Pantomimen - Filmszenarien, S. 482.

200 Mathias Mayer 
enthaltes im Jahr 1900 hat Hofmannsthal, in Begleitung seines späteren Schwagers, des Malers Hans Schlesinger, den "weiten Weg ins Faubourg St. Germain" angetreten. ${ }^{10}$ Hans Schlesinger studierte seit 1894 an der Académie Julian im Montmartre-Viertel Malerei und hatte Hofmannsthal wiederholt nach Paris eingeladen. ${ }^{11}$ Über Hans Schlesinger ist er im Frühjahr 1900 mit der Aristokratie in Verbindung gekommen - dieser brachte Hofmannsthal u.a. zur Baronin Deslandes, der kunstsinnigen Sammlerin, die Edward Burne-Jones porträtiert hat, ${ }^{12}$ die ihn »nächstens mit Montesquiou « einladen wollte, wie er den Eltern am 25. Februar 1900 schrieb. $^{13}$ Auf diese Weise lernte Hofmannsthal gleich mehrere "Originale" der Proustschen Romanwelt kennen, ausgerechnet in Montesquiou sollte er einer Person begegnen, deren fiktive Verwandlung als Baron Charlus ihn 20 Jahre später ebenso fesseln wie inspirieren sollte denn seine Lektüre von Prousts Roman führte dazu, dass er Charlus als Modell für die Figur des Maltesers im "Andreas«-Roman reklamierte, diese Proustsche Figur aber auch wieder mit Erfahrungen persönlicher Betroffenheit verknüpfte: In der Notiz 223 hält Hofmannsthal auf bemerkenswerte Weise einen Zusammenhang fest, der die bei Proust in Gang gekommene Durchlässigkeit von Person und Figur weiterführt. "Der Malteser. Synthese. Anklänge an welche Geister? Kessler George Pannwitz Stendhal Charlus « ${ }^{14}$. In weiteren Notizen vertieft Hofmannsthal diese Linien und zitiert sogar eine Passage aus "Sodome et Gomorrhe« ${ }^{15}$ zur Charakterisierung des Barons. Dieser Band ist in Hofmannsthals Nachlassbibliothek nicht mehr erhalten, wohl aber die Teile »Du côté de chez Swann« (33. Aufl. 1919), "La Prisonnière« (31. Aufl. 1924) und »Le Temps rerouvé« (13. Aufl. 1927). In "La Prisonnière« hat Hofmannsthal eine Passage angestrichen, aber auch Werknotizen zu dem Plan »Das Hotel« von 1929 festgehalten, die von der Proustlektüre offensichtlich

10 B II, S. 16.

11 Vgl. dazu die erhellende Darstellung von Katja Kaluga, Paris. Die größere Welt. In: Hofmannsthal. Orte. 20 biographische Erkundungen. Hg. von Wilhelm Hemecker und Konrad Heumann. Wien 2014, S. 176-199, hier S. 178.

12 Ebd., S. 190 f.

13 B II, S. 14.

14 SW XXX Roman, S. 161.

15 Vgl. ebd., S. $201 \mathrm{f}$. 
beeinflusst sind, so u.a. "Die `Dame mit dem Vermeer « ${ }^{16}$ ferner indem das Thema der lesbischen Liebe aufgegriffen wird. ${ }^{17}$

Vermutlich war es dem Proustleser Hofmannsthal nicht bewusst, dass er einige der von ihm verwendeten Modelle selbst persönlich kennengelernt hatte, so etwa den Schriftsteller Anatol France, der mit einigen Zügen in die Figur Bergottes einging (der in "La Prisonnière" bekanntlich vor dem Anblick eines Vermeer-Gemäldes tot zusammenbricht), oder aber die große Dame der Pariser Gesellschaft, die eines der Urbilder der Herzogin von Guermantes werden sollte: Aus Paris hatte Hofmannsthal im Februar/März 1900 geschrieben, er habe während eines Botschaftsempfangs "ein bißchen mit der berühmten, sehr schönen Gräfin Greffulhe (geb. Chimay) gesprochen «. ${ }^{18}$ Gegenüber dem Grafen Kessler heißt es am 29. März 1900 über die »Frauen der Gesellschaft«, darunter auch Gräfin Greffulhe, sie ließen ihn »nicht ohne eine gewisse Enttäuschung und Ungeduld «. ${ }^{19}$

Vor allem aber kommt ein weiterer Umstand hinzu: Dass Hofmannsthal während seines Parisaufenthaltes mit Proust selbst in Verbindung gekommen wäre, lässt sich nicht belegen, so reizvoll die Vorstellung ist, sie wären gemeinsam mit Montesquiou und der Gräfin auf einem Empfang gewesen. Eine Schlüsselrolle kommt dabei Hans Schlesinger zu, der aber Proust vermutlich erst kurz danach kennengelernt hat, denn am 16. Mai 1900 schreibt er aus Venedig an Hofmannsthal: "Es ist alle mögliche Gesellschaft im Hôtel - ein gewisser Marcel Proust «. ${ }^{20}$ Aber Hans Schlesinger taucht im Umkreis Prousts denn doch noch weiter auf: In Versailles kam es im August 1906 zu einer kuriosen Begegnung, bei der Proust "Schlesinger « zunächst nicht wiedererkannte, sondern ihn mit dem alkoholisierten Ex-Diener seines Vaters verwechselte, dann aber von Schlesinger erfuhr, dass sie Wand an Wand wohnten. Der Maler machte Proust daraufhin Komplimente über seinen Bart, die indes durch den Hinweis auf das dadurch kaschierte Älterwerden für Proust zweifelhaft gewesen sein müssen: »Il m'a dit que ma barbe m'allait très bien

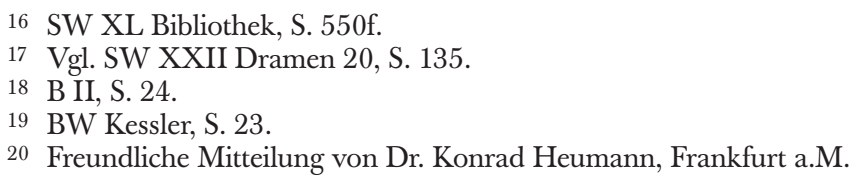


scar cela va toujours bien aux figures vieilles et vieillies«", wie Proust an Reynaldo Hahn schrieb, der Schlesinger besser kannte. ${ }^{21}$

Eine persönliche Begegnung zwischen Proust und Hofmannsthal ist nur über Dritte bezeugt, Christiane Zimmer vergewisserte ihrem Interviewpartner Horst Weber, ihr Vater »kannte zum Beispiel Marcel Proust sehr gut «. ${ }^{22}$ Und Harry Graf Kessler hält in seiner Erinnerung an die Entstehung der "Josephslegende« fest, Ende Mai/Anfang Juni 1912 sei es in Paris zu einem Abend mit Proust gekommen, der "ganz in dicke Halstücher eingemummt" gewesen sei. ${ }^{23}$ Als Hofmannsthal im März 1913 überlegte, dem stellenlos gewordenen Dramaturgen Karl Freiherr von Gersdorff (1866-1914) eine Alternative anzubieten, schlug er in einem Brief an Ottonie Gräfin Degenfeld vor, sich u.a. an die Königin von Neapel zu wenden - was die Herausgeber des Briefwechsels als eine "nicht mehr verständliche Anspielung « kommentierten. ${ }^{24}$

Hofmannsthal hat somit die Durchlässigkeit der Grenzen zwischen Lebenswelt und Literatur im Umkreis Prousts aus beiden Perspektiven verfolgen können - die Sonderrolle der Königin von Neapel scheint er indes bei seiner Proustlektüre nicht wahrgenommen zu haben. Dass diese für ihn bedeutsam war, zeigen neben den schon genannten Verbindungen in eigene Werke auch die Briefzeugnisse. 1922 schrieb er an Leopold von Andrian, beim "Hineinschauen in das Buch von Proust « habe er »bemerkt[,] wie sehr mich dasselbe beim Verfertigen eines neuen Lustspiels (also im Spätsommer) anregen könnte«. ${ }^{25}$ Und im Jahr darauf empfahl er Ottonie Degenfeld die beiden ersten Teile der "Recherche«: "Es hat nie jemand - oder mindestens seit dem XVIIten Jahrhundert nicht - dies Phänomen `die Gesellschaft‘ so gesehen und so etwas daraus gemacht «. ${ }^{26}$

Das mögen historische Zufälle oder vielleicht auch Konstellationen oder Sternstunden gewesen sein, in denen jedenfalls eine europäische Koope-

21 Marcel Proust, Correspondance. Texte établi, présenté et annoté par Philip Kolb. Bd. VI: 1906. Plon 1980, S. 186; vgl. Painter, Proust 2 (wie Anm. 5), S. 99 f.

22 Freundlicher Hinweis von Dr. Konrad Heumann, Frankfurt a.M.

23 Harry Graf Kessler, Künstler und Nationen. Aufsätze und Reden 1899-1933. Frankfurt a.M. 1988, S. 277. - Leider ist diese Begegnung in Kesslers Tagebuch nicht festgehalten.

24 BW Degenfeld (1986), S. 259 und 590.

25 BW Andrian, S. 331.

26 BW Degenfeld (1986), S. 470f. 
ration und Solidarität noch praktiziert wurde - das Ballett einer deutschösterreichischen Autorschaft wird in Paris von den »ballets russes« aufgeführt. Sehr viel entscheidender ist der Stellenwert der kleinen Nebenfigur der Königin von Neapel in Prousts riesigem Pandämonium. Dazu kann ein nicht weniger erstaunliches Dokument herangezogen werden, denn die Königin von Neapel hat, offenbar in Deutschland, kurze Zeit vor ihrem Tod eine Lesung aus der "Gefangenen« verfolgt - und über die zitierte Szene gesagt: »Es ist seltsam, ich habe diesen Monsieur Proust nie gekannt, aber er scheint mich sehr gut gekannt zu haben, denn er läßt mich so handeln, wie ich es meiner Ansicht nach getan hätte«. ${ }^{27}$ Aus der Biographie von Tadié ist ersichtlich, woher Proust seine Kenntnisse der Königin hatte: Er war mit Illan de Casa-Fuerte (1882-1962) befreundet, dessen spanischer Vater als Ehrenattaché der spanischen Gesandtschaft die Revolution in Neapel im Jahr 1860 miterlebt hatte. Prousts Freund hat über diese Vorgänge auch in seiner Autobiographie berichtet. ${ }^{28}$ Proust, so wäre zu überlegen, hat in diesem Fall historisch-biographische Erfahrungen ausnahmsweise unverschlüsselt in seinen Text aufgenommen. Er hat damit einer Person ein Denkmal gesetzt, ohne dass es zwischen ihnen einen persönlichen, in beide Richtungen ausstrahlenden Kontakt gegeben hätte, denn sie kannte ihn nicht. Mit dieser Episode rührt Proust an eine im Roman zuletzt intensiv reflektierte Schicht, die über die "wiedergefundene Zeit» des Erzählens hinausgeht. Der Roman öffnet sich in dieser bemerkenswerten Episode über die erinnerte Zeit in eine erfundene Szene, die in der Logik des Textes zwischen Realität (Königin von Neapel) und Fiktion (Charlus) oszilliert, die aber gerade darin zu einer Erfahrung der Leserin/des Lesers werden kann. Genau darüber wird der Erzähler in der Schlüsselszene des gesamten Romans nachdenken, in der Szene in der Bibliothek des Prinzen von Guermantes. Dort entwickelt er jene gänzlich antirealistische Literaturtheorie, wonach es nicht um exakte Beschreibung geht, nicht um platten oder direkten Biografismus, sondern um den Weg zu den Tiefen, »in denen das, was wirklich existiert hat, von uns ungekannt ruht« (Bd. VII, 298). Vielleicht ist es gerade der durch die Königin von Neapel erprobte Grenzbereich zwischen Realität und Fiktion, der jene Perspektive des Romans beson-

27 Painter, Proust 2 (wie Anm. 5), S. 392.

28 Tadié, Proust (wie Anm. 5), S. 501-504; Illan de Casa-Fuerte, Le Dermier des Guermantes. Paris 1995. 
ders beglaubigen kann, die ebenfalls in der großen Bibliotheksszene, beim Nachdenken über die Literatur als das wahre Leben, formuliert wird: "In Wirklichkeit ist jeder Leser, wenn er liest, ein Leser nur seiner selbst. Das Werk des Schriftstellers ist dabei lediglich eine Art von optischem Instrument, das der Autor dem Leser reicht, damit er erkennen möge, was er in sich selbst vielleicht sonst nicht hätte erschauen können« (Bd. VII, 318).

Kann man diese Figur, wie der Verdacht nahelegt, den Biografen und Anekdotenjägern überlassen? Zwar hat man sich in den Personallexika der Proustforschung über die historische Authentizität der biografischen Angaben verständigt, aber, wie es scheint, hat man wenig Anlass gesehen zu der Frage, wie es dazu kommt, dass Proust in diesem Fall, mag er auch nicht der einzige sein, so ist er doch prominent genug!, von dem Verfahren der Verschlüsselung und Amalgamierung mehrerer historisch(auto-)biografischer Personen zu einer literarischen Figur abgewichen ist? Gerade wenn es der Fall sein sollte, dass die Königin von Neapel als eine Ausnahme in diesem Werk beschrieben werden kann, wäre es umso dringlicher, die damit zumindest suspendierte Regel genauer zu befragen und den Wert der Ausnahme zu beschreiben. Somit könnte sehr viel dafür sprechen, diese Romanfigur gerade nicht der schlichten Biografik und Anekdotenforschung zu überlassen, zumindest wenn von dieser Ausnahme her Einblicke in die Struktur des Proustschen Werkes möglich würden. Ein Argument könnte dabei sein, dass der Grenzbereich, den sie verkörpert - nämlich zugleich historische Person (mit biografischen Daten) zu sein und überdies fiktive Figur eines Romans - einen Aufschluss gibt über Prousts freilich extrem komplexes Verständnis »realistischer Literatur. ${ }^{29}$ In diesem Zusammenhang bietet es sich an, an seine Auseinandersetzung mit der »Methode Sainte Beuves« zu erinnern, die ja eine der ästhetisch-kritischen Voraussetzungen der "Recherche« bildet. In der eher programmatisch gehaltenen Diskussion von dessen Literaturverständnis hält Proust fest, für Sainte-Beuve sei es von grund-

29 Hilfreiche Expeditionen in die »indeterminay" zwischen Fiktion und Autobiografie unternimmt Dorrit Cohns Aufsatz "Proust's Generic Ambiguity" in ihrem Buch »The Distinction of Fiction" (Baltimore/London 1999, S. 58-78, hier S. 59 und 64). Sie beobachtet »illusionist games« (S. 65) und "a generally hybrid creature« (S. 67). Vgl. auch Frank Jäger, Literarische Selbstinszenierung zwischen Transgression und Paradoxie. Zur Hybridisierung autobiographischen Schreibens bei Marcel Proust, Michel Leiris und Claude Simon. Freiburg i.Br. 2014. 
legendem Interesse, einen Autor aus seinem Lebenszusammenhang zu studieren, dass man sich in Bezug auf ihn

eine bestimmte Anzahl von Fragen gestellt und sie beantwortet hat, und sei es nur leise und für sich ganz allein [...]: Was dachte er über die Religion? Wie berührte ihn das Schauspiel der Natur? Wie verhielt er sich in bezug auf die Frauen, in bezug auf das Geld? War er reich oder arm? Welches war seine Lebensordnung, seine alltägliche Lebensweise? Welches war sein Laster oder seine Schwäche? Keine Antwort auf diese Fragen ist unwichtig,

so resümiert Proust den Standpunkt seines Gegners, um ihm dann die eigene Auffassung entgegenzustellen: Diese Befragung der persönlichen und geistigen Umgebung, für die Sainte-Beuve so viel Anerkennung erfahren hat (u.a. von Taine und Paul Bourget), sei falsch, sie

verkennt, was ein etwas tieferer Umgang mit uns selbst uns lehrt: daß ein Buch die Hervorbringung eines anderen Ichs ist als dessen, das wir in unseren Gewohnheiten, in der Gesellschaft, in unseren Lastern zutagetreten lassen. Wenn wir versuchen wollen, dieses Ich zu verstehen, so kann uns das nur im Innersten von uns selbst gelingen, indem wir versuchen, es in uns nachzuschaffen $[\ldots]^{30}$

Was besagt das über die Königin von Neapel? So viel, dass es auch im Fall einer historischen Figur nicht das Interesse am Historisch-Realen gewesen sein dürfte, das ihr den immerhin spektakulären Platz in der Matinee der Verdurins zuspricht. Proust unterbricht ihretwegen nicht den Charakter der Gesellschaftsschilderung, sondern das ganze - in diesem Ausnahmefall ja stimmige historische - Material, das mit ihr und ihrer Vorgeschichte aufgefahren wird, interessiert ausschließlich, und gut getarnt, als Teil der Romanfiktion. Man nimmt die Königin von Neapel als selbstverständlich glaubhaften Teil des aristokratischen Panoramas wahr, d.h., ein bloß realistisches Interesse ist gerade nicht relevant.

Insofern sollte die Episode der Königin von Neapel nicht einfach in die Balzac-Nähe Prousts gerückt werden, auch wenn bereits eine Fülle "verborgene[r] Mikro-Balzac-Pastiches" bei Proust rekonstruiert wurde. ${ }^{31}$ Bei Balzac, den Proust intensiv rezipiert hat und mit den fiktiven

30 Marcel Proust, Die Methode Sainte-Beuves. In: Ders., Gegen Sainte-Beuve. Deutsch von Helmut Scheffel. Frankfurt a.M. 1962, S. 7-39, hier S. 13f.

31 Francine Goujon, Balzac I. In: Luzius Keller (Hg.), Marcel Proust Enzyklopädie. Handbuch zu Leben, Werk, Wirkung und Deutung. Hamburg 2009, S. 76 - 78, hier S. 78. 
Lesern Charlus und dem Herzog von Guermantes verknüpft, findet sich bereits die Praxis, historische und fiktive Namen zu mischen. Darüber hat Proust im Balzac-Kapitel von "Contre Sainte-Beuve« geschrieben, indem er ihn von Flaubert absetzt, denn im Unterschied zu diesem habe Balzac »die im Leben errungenen Triumphe völlig auf die gleiche Ebene [gestellt] mit denen der Literatur ${ }^{32}{ }^{32}$ \#s gab«, so Prousts Urteil weiter, »keine Trennungslinie zwischen dem wirklichen Leben (dem, was es unserer Meinung nach nicht ist) und dem Leben in seinem Roman (dem einzig wahren für den Schriftsteller)« ${ }^{33}$

Wie sehr Balzac damit ein poetisches Verfahren propagiert, von dem sich Prousts Umgang mit historischen Gestalten unterscheidet, geht aus einer genaueren Reflexion hervor, in der er Balzacs Technik als eine Art Diskreditierung der Kunst entlarvt:

Es ist keine reine Illusion, wenn Balzac, in der Absicht, Namen großer Ärzte oder großer Künstler zu zitieren, in völligem Durcheinander wirkliche Namen und Personen aus seinen Büchern nennt und sagt: »Er hatte das Genie eines Claude Bernard, eines Bichat, eines Despleins, eines Bianchon«, wie jene Maler von Panoramen, die im Vordergrund ihrer Werke vollplastische Figuren und perspektivische Täuschungen des Dekors miteinander vermengen. Sehr oft sind diese wirklichen Personen nichts weiter als wirklich. Das Leben dieser Personen ist eine Wirkung der Kunst Balzacs, verschafft aber dem Autor eine Befriedigung, die nicht in den Bereich der Kunst gehört. ${ }^{34}$

Gerade an dieser zu offensichtlichen Demonstration des Realistischen hat Proust kein Interesse - "seine« Königin von Neapel soll nicht dem Autor eine kunstfremde Befriedigung schaffen, als ein Relikt des wirklichen Lebens, das ins Werk eingearbeitet wird. Proust propagiert im Gegenteil die Unsichtbarmachung des realen Lebens, um auf diese Weise die "reine Illusion" zu erzeugen. Wie sehr es ihm auf diese kunstgerechte Handhabung ankommt, als Verwandlung von Lebensstoff in die Kathedrale seines Textes, zeigt sich nirgendwo so deutlich wie dort, wo die Gefahr der Balzacschen Realitätsnähe so groß war. Tadié verweist überdies auf die historischen Anekdoten, die Proust bei Georges Lenôtre (18571935) kennengelernt hatte: Er hatte »immensen Erfolg mit seiner $>\mathrm{Ge}-$

32 Marcel Proust, Sainte-Beuve und Balzac. In: Ders., Gegen Sainte-Beuve (wie Anm. 30), S. 96-137, hier S. 99.

33 Ebd., S. 101.

34 Ebd., S. 107f.

Marcel Prousts Königin von Neapel 207 
schichte im kleinens: mit der Geschichte von zweitrangigen Figuren, mit Anekdoten und Sittenbildern aus der Zeit der Revolution und des Ersten Kaiserreichs. Solche unauffälligen Figuren ähneln Romanhelden. $\aleph^{35}$ Lenôtre hatte seinerseits wieder in Hofmannsthal einen anderen interessierten Leser gefunden. ${ }^{36}$

Ein »Fall« aus der Hocharistokratie, der der Königin von Neapel am nächsten kommen mag, ist die Prinzessin Mathilde (1820-1904), in deren Salon Proust seit 1891 verkehrt hatte. ${ }^{37}$ Swann stellt sie dem Erzähler bei einer Begegnung in der Nähe des zoologischen Gartens vor, mit dem Kommentar: "Sie wissen ja die Freundin von Flaubert, von Sainte Beuve, von Dumas. Stellen Sie sich vor, sie ist die Nichte Napoleons I.! Napoleon III. und der Kaiser von Rußland haben um sie geworben. Ist das nicht interessant?" (Bd. II, 153f.) Auch hier also: Eine historische Gestalt, die im Dialog mit den Figuren des Romans gezeigt wird und ihnen somit, wie es scheinen könnte, einen Anschein von Realität stiften könnte? Der Fall liegt doch wohl eher umgekehrt: Gerade angesichts der Möglichkeit, historische Gestalten für eine Entfiktionalisierung zu nutzen und somit der vermeintlichen »Wirklichkeit» der Daten, der Geschichte zum Einzug in den Roman zu verhelfen, entscheidet sich Proust dafür, Elemente der historischen Realität geradezu unscheinbar zu integrieren, um die eigentliche Substanz der gelebten Wirklichkeit stärker hervorzuheben. Im Fall der Prinzessin Mathilde ist dieses Verfahren dadurch erleichtert, dass Prousts Schilderung seiner Besuche in ihrem Salon als Chronik im »Figaro" erschien (25. Februar 1903): "Ein historischer Salon. Der Salon ihrer Kaiserlichen Hoheit Prinzessin Mathilde « ${ }^{38}$, dass aber die Übertragung der Gestalt in die Welt des Romans (in "À l'ombre des jeunes filles en fleurs«) erst 1919 publiziert wurde (Prinzessin Mathilde war 1904 verstorben). Im Fall der sehr viel riskanteren und zugleich bewegenderen Szene mit der Königin von Neapel hat Proust zu deren Lebzeiten ihre Fiktionalisierung betrieben.

Doch ist dies bei Proust umso erstaunlicher, als er ganz bewusst eine Gelegenheit ausschlägt, das realistische Fundament hinter seinem Text durchschimmern zu lassen. Man könnte vielmehr zu der Beobachtung

35 Tadié, Proust (wie Anm. 5), S. 516.

36 SW XL Bibliothek. S. $426 f$.

37 Vgl. Painter, Proust 1 (wie Anm. 5), S. $156 f$.

38 Ebd., S. 477f.

208 Mathias Mayer 
kommen, dass gerade die Irrelevanz des Realistischen/Biografischen/ Historischen für den besonderen Charakter der Figur spricht. Sie würde, eben als Grenzfall, zu einem Paradigma jenes Teils von Prousts Ästhetik, der sich, über die Kritik an Sainte-Beuves Methode hinaus, als Einspruch und Verweigerung realistischen Erzählens beschreiben ließe. Eine der prominentesten Passagen zu diesem Komplex findet sich in jener Schlüsselszene des Romans - dem Aufenthalt des Erzählers in der Bibliothek des Prinzen von Guermantes im Zentrum der »Wiedergefundenen Zeit«. Hier formuliert der Erzähler ein grundlegendes Misstrauen gegen die Naivität einer unmittelbar gegebenen und damit widerzuspiegelnden Wirklichkeit.

Was wir die Wirklichkeit nennen, ist eine bestimmte Beziehung zwischen Empfindungen und Erinnerungen, die uns gleichzeitig umgeben - eine Beziehung, die bei einer einfachen kinematographischen Wiedergabe verlorengehen würde, da diese sich um so mehr von der Wahrheit entfernt, je mehr sie sich auf sie zu beschränken vorgibt - eine einzigartige Beziehung, die der Schriftsteller wiederfinden muß, um für immer in seinem Satz die beiden verschiedenen Pole miteinander zu verbinden (Bd. VII, 288f.).

Eine »bloß aufzeichnende Literatur" (Bd. VII, 296) ist ohne Wert, denn sie versäumt es gerade,

jene Wirklichkeit, von der wir so weit entfernt leben, wiederzufinden, wieder zu erfassen und uns bekanntzugeben, die Wirklichkeit, von der wir uns [in realistischer Literatur] immer mehr entfernen, je mehr die konventionelle Kenntnis, die wir an ihre Stelle setzen, an Dichte und Undurchdringlichkeit gewinnt, jene Wirklichkeit, deren wahre Kenntnis wir vielleicht bis zu unserem Tode versäumen und die doch ganz einfach unser Leben ist (Bd. VII, 297).

Sicherlich, hier ist die Rede von dem antirealistischen Schreiben, das das "einzig wahre Buch, da es bereits in jedem von uns existiert, nicht im landläufigen Sinne erfinden, sondern übersetzen muß« (Bd. VII, 290) und die Königin von Neapel ist als Figur eines solchen Schreibens auf einer anderen Ebene angesiedelt. Aber sie dient darin als poetologische Ikone der Irrelevanz des Realen und des Realistischen, denn nicht ihre "Echtheit«, nicht das kinematografische Abbild, sondern die Verwischung dieses realen Hintergrundes macht sie, von einer Seite, zu einem entscheidenden Instrument des Proustschen Schreibens. 
Ein weiteres Argument müsste man ebenfalls prüfen, das in die Tiefen der Entstehungsgeschichte des Romans zurückreicht, und vielleicht in autobiografische Verletzungen des Protagonisten. Wie schon George D. Painter im zweiten Band seiner Biografie dargelegt hat, gibt es ein Vorbild für die Szene der Solidarität zwischen Charlus und der Königin von Neapel in dem wohl 1895 begonnenen Roman "Jean Santeuil«, der allerdings Fragment blieb. In dessen »kläglichsten Kapiteln", wie der Biograf vermeint, wird indes nicht ein Dritter, sondern der Protagonist selbst von Madame Marmet beleidigt - einer Vorläuferfigur für Madame Verdurin - und von der mutigen Geste der Herzogin von Réveillon unterstützt. ${ }^{39}$ Die Genugtuung nach öffentlich erfolgter Demütigung und Beleidigung wird bereits hier in der Geste der Unterstützung dargestellt: "Mein lieber Jean«, sagt die Herzogin von Réveillon, indem sie sich vom Arm des Herzogs von Litauen löst, "Sie sind zur Zeit so leidend und brauchen zu Ihrer Stütze gewiß einen stärkeren Arm als den von Madame Cygnerolles. ${ }^{40}$ Bei dieser fiktiven Figur handelt es sich um eine Vorläufergestalt der Herzogin von Guermantes ${ }^{41}$ wenngleich der reale Besitz "Réveillon" Madelaine Lemaire gehörte und daher Proust aus verschiedenen Aufenthalten und Spaziergängen gut bekannt war: Aber auch hier sollte gar nicht die biografische Fundierung im Mittelpunkt des Interesses stehen, sondern die erstaunliche Tatsache, dass Proust eine Szene der Solidarität in mehrfacher Weise verschoben oder umgekehrt hat: 1. Von der Figur des stark autobiografisch geprägten Jean Santeuil zu der des Baron Charlus, »der eigentlichen Zentralfigur der Recherche", wie Adorno meint. ${ }^{42}$ 2. Von einer Romanfigur (Herzogin von Réveillon) auf eine historische Gestalt (Königin von Neapel). Diese Doppelverschiebung kann grafisch als ein Chiasmus dargestellt werden.

$\begin{array}{llll} & \text { autobiografisch/historisch } & & \text { fiktiv } \\ \text { Degradierung: } & \text { Jean Santeuil } & -> & \text { Charlus } \\ \text { Solidarität: } & \text { Königin von Neapel } & <- & \text { Herzogin von Réveillon }\end{array}$

39 Ebd., Bd. 2, S. 375, 392.

40 Marcel Proust, Jean Santeuil. Deutsch von Eva Rechel-Mertens. 2 Bde. Frankfurt a.M. 1984, Bd. 2, S. 291.

41 Painter, Proust 2 (wie Anm. 5), S. 122.

42 Adorno, Kleine Proust-Kommentare (wie Anm. 1), S. 211. 
Offenbar geht es also um eine Art Osmose zwischen den Ebenen, denn indem die Peinlichkeit der Degradierung weggeschoben wird vom autobiografisch-real erlebten Moment hin zu einer fiktiven Szene, gewinnt die umgekehrt entsprechende Bewegung der Solidarität und Anerkennung ihr Gewicht dadurch, dass sie von einer fiktiven Figur auf eine historische Gestalt übertragen wird. Dass mit dieser Vertauschung indes eine Aufwertung der Szene verknüpft ist, zeigt sich in der höchst erstaunlichen, bewegenden Tatsache, dass Proust bei der Szene in der »Gefangenen« die seltene Entscheidung getroffen hat, eine nichtfiktive Figur in ein fiktives Universum einzuschmuggeln. Indem aus der Herzogin von Réveillon die Königin von Neapel wird, gehen die Schutz- und Maskierungsmaßnahmen verloren, die Proust in der Regel bei seinen Romanfiguren, wie bekannt, sonst walten lässt. Als Königin von Neapel ist die Figur sozusagen, zumal in der ausführlichen Darstellung, wie sie in der zitierten Passage geboten wird, in historischer Unmittelbarkeit riskiert die hohe Dame der Gesellschaft tritt maskenlos in den Roman ein, aber die entfiktionalisierende Benennung des historischen Namens führt statt in eine peinliche Entlarvung in eine höchst überzeugende Aufwertung, in Respekt und Anerkennung. Deshalb scheint die Randfigur und Ausnahmegestalt der Königin von Neapel mehr als nur ein Kuriosum, mehr als nur eine Anekdote zu sein: Sie bietet eine geradezu unerfindbare Authentizität schlichter Lebenswahrheit, auf die es Proust angekommen ist. Auch wenn wir nicht wissen, welche Begegnung zwischen dem Autor und der Königin von Neapel stattgefunden haben mag - jenseits des Biografischen vermittelt die Szene der »Gefangenen« einen Schlüssel für die Durchlässigkeit von Realität und Fiktion, für die Erkenntnis- und Fiktionsproblematik von Prousts Schreiben.

Schließlich wäre noch daran zu denken, inwiefern die Königin von $\mathrm{Ne}$ apel für Proust etwas von der Dimension angenommen hat, die ihn an den Romanen Dostojewskijs so fasziniert hat: Im November 1920 hatte er dem Verleger gegenüber davon gesprochen, „Le Côté de Guermantes" sei "auf eine [...] mehr Dostojewskij'sche Weise gestaltet« ${ }^{43}$ und der im September 1921 ins Gespräch gebrachte Essay über Dostojewskij] ${ }^{44}$ findet sich schließlich als Reflexion des Erzählers am Ende der »Gefange-

43 Proust, Briefe zum Werk (wie Anm. 2), S. 424.

44 Vgl. ebd., S. 453.

Marcel Prousts Königin von Neapel 211 
nen«, wenn der Erzähler Albertines Klavierspiel unterbricht. Anhand des "Idioten« und der »Brüder Karamasov" erörtert er die Frauengestalten "mit ihrem geheimnisvollen Antlitz, dessen ansprechende Schönheit jäh, als habe sie nur die Komödie der Güte aufgeführt, zu furchtbarer Anmaßung erstarrt" (Bd. V, 507). Albertine ihrerseits, eine versierte Leserin, wie sich zeigt, greift die Argumentation auf, wenn sie beobachtet: »Wir sind alle erstaunt, hinterher zu erfahren, daß dieser oder jener heimtückische Mensch im Grunde ein ausgezeichneter Charakter ist, oder umgekehrt« (Bd. V, 509). Diese Erfahrung einer nicht absehbaren Umkehrung kann bei der Königin von Neapel festgestellt werden, wenn auch weniger in einer moralischen Perspektive: Sie wird am Abend bei den Verdurins als eine Gestalt der Hocharistokratie eingeführt, als Schwester der Kaiserin Elisabeth (Bd. V, 328), aber es zeigt sich, dass sie zugleich ohne Macht und ohne Dienerschaft ist, wie Charlus zunächst hämisch "mit der Absicht ziemlich massiver Ironie« insinuiert: "Bedenken Sie, daß die Königin von Neapel zum Zweck der Teilnahme an Ihrem Fest aus Neuilly gekommen ist, was sehr viel schwieriger für sie war, als die Beiden Sizilien zu verlassen" (Bd. V, 366). Aber eben in der von ihrer Entmachtung gezeichneten Schlichtheit wird sie kurz darauf - und das wäre ein Umschwung im Sinne Dostojewskijs - eine allen gesellschaftlichen Dünkel überstrahlende Größe beweisen, die sich als Güte erweist, mit der sie eben nicht nur ihresgleichen (wie Charlus) begegnet, sondern auch »Menschen des Bürgertums oder auch des einfachsten Volkes, welche die zu achten wußten, die sie selber liebte, und für sie gute Gefühle hegten« (Bd. V, 431).

Indem Proust ihr eine solche Menschlichkeit zuspricht, holt er eine Wahrheit des Lebens ans Licht, die nicht auf Standesgrenzen angewiesen ist - im Gegensatz zu Madame Verdurin, die freilich in dritter Ehe noch selbst zur Prinzessin von Guermantes aufsteigen kann (Bd. VII, 378) - und die daher in einem bezeichnenden und vielleicht sogar einmaligen Licht zwischen Realität und Fiktion zu stehen kommt. 